\title{
A Mobile-Based Prototype for Skin Lesion Analysis: Towards a Patient-Oriented Design Approach
}

\author{
http://dx.doi.org/10.3991/ijoe.v9iS8.3372 \\ Luís Rosado $^{1}$, Maria João Vasconcelos ${ }^{1}$ and Márcia Ferreira ${ }^{2}$ \\ ${ }^{1}$ Fraunhofer Portugal AICOS, Porto, Portugal \\ 2 Portuguese Institute of Oncology, Porto, Portugal
}

\begin{abstract}
The wide spreading of the new generation of smartphones, with significant improvements in terms of image acquisition and processing power, is opening up the possibility of new approaches for skin lesion monitoring. Mobile Teledermatology appears nowadays as a promising tool with the potential to empower patients to adopt an active role in managing their own skin health status, while facilitates the early diagnosis of skin cancers. The main objective of this work is to create a mobile-based prototype for skin lesions analysis with patient-oriented features and functionalities. The presented self-monitoring system collects, processes and storages information of skin lesions through the automatic extraction and classification of specific visual features. The algorithms used to extract and classify these features are briefly described, as well as the overall system architecture and functionalities.
\end{abstract}

Index Terms-Melanoma Detection, Mobile Teledermatology, Mobile Monitoring, Supervised Classification.

\section{INTRODUCTION}

Skin cancers constitute nowadays one of the most common malignancies in the Caucasian population, with incidences that are reaching epidemic proportions [1]. The importance of early diagnosis in malignant melanoma cannot be overstated, since the success rates of curing this type of cancer are very high if detected during the early stages of its development. Another relevant motivational factor for this work is the inadequate distribution of dermatologists in Portugal. A recent study [2] showed that this issue comes from two main factors: (1) clear uneven regional distribution of dermatologists, with over allocation of specialists near the big urban centers; (2) the number of dermatologists currently working on the healthcare system represents only $60 \%$ of the required resources estimated. During the last decades several diagnostic algorithms have been proposed, being the $\mathrm{ABCD}$ rule one of the most widely accepted method. This rule was proposed in 1994 [3] and is based on the analysis of 4 different criteria: asymmetry (A), border (B), color (C) and differential structures (D). Thus, Mobile Teledermatology (MT) appears as a promising tool for personal dermatology data acquisition [4], [5], with the potential of becoming an easy applicable tool that empowers patients to adopt an active role in managing their own health status, facilitating the early diagnosis of skin cancers, and offering the opportunity to bring dermatological expertise into underserved areas.

\section{SYSTEM DESIGN APPROACHES}

In this chapter two different design approaches for skin lesion analysis systems are described: the doctororiented and the patient-oriented design approaches.

\section{A. Doctor-Oriented Design}

In recent years, skin lesion analysis has been a hot topic in the computer vision research field. The great majority of the proposed methodologies on the literature are based on dermoscopic image analysis, usually aiming for dermatology specialist's usage as decision support systems. Despite high accuracies for melanoma detection being stated in recent publications [6], there's a technology maturation still needed for the deployment of this type of technologies in the clinical practice. Taking also into account the significant skepticism of dermatology specialists in relying on automatic image analysis for risk assessment purposes, doctors that will potentially use these approaches in the near future will certainly not discard conducting their own visual examination. This limitation confers to these approaches only a slim added value for the doctor, being one of the biggest challenges nowadays for their deployment in the clinical practice.

\section{B. Patient-Oriented Design}

From a patient perspective, one can identify different needs and implementation strategies. Patients with clinical or family history of skin cancer should consult regularly their dermatologists for physical skin examinations, for instance once a year. Between these appointments the patients are usually advised by their doctors to check for relevant changes in their skin moles, and anticipate the consultation in case of detecting something suspicious. But unfortunately these patients typically don't have enough dermatological expertise to do this kind of risk assessment. The patient-oriented approaches are new paradigms for skin lesion analysis that are trying not only to motivate and educate the patients, but also to empower them in terms of dermatological expertise, which can lead to a significant impact in the early diagnosis of skin moles malignancies. The goal of this work is to explore the design of a mobile-based system for skin lesions analysis with patient-oriented features and its functionalities.

\section{SYSTEM ARCHITECTURE}

The architecture of the proposed patient-oriented system is formed by 3 main blocks: 
1. Front-end Device (The user's smartphone): used to acquire or load an image of a skin lesion and send it to the server for analysis. The application that allows the communication between the smartphone and the server was developed in Android 4.0, using the HTTP communication protocol.

2. Server: The server side consists on a RESTFul webservice implemented in Java using the Jersey library [7] and deployed on an Apache Tomcat $7.0 \mathrm{web}$ server running on Ubuntu 10.04. The Image Processing Module (IPM) was implemented in C++ using the OpenCv library [8], which is executed as an external program when an image is received in the server. The IPM receives as input the original skin lesion image and returns a quantitative analysis of the image in addition to a visual output of the segmentation and features extraction steps.

3. Back-end Device: The device that will receive the output images generated by the IPM analysis. In the current version of the prototype the results are only returned to the user's smartphone; although considering that the IPM analysis information can also be provided to dermatology specialists, a web interface is currently being developed.

\section{IMAge Processing Methodology}

The IPM comprises 5 steps: pre-processing, segmentation, feature extraction, feature selection and supervised classification.

\section{A. Pre-processing}

Two pre-processing steps were implemented in order to ensure a good segmentation of the images: (a) Circular Averaging Filtering using a disk-shaped structuring element of radius 5; and (b) Automatic Color Equalization: a technique proposed by Schaefer et al. [9] was used for contrast enhancement.

\section{B. Segmentation}

The algorithm used for lesion segmentation was based on the Otsu's Method [10], a well-known histogram shape-based image thresholding routine which assumes that the input image has two classes of pixels, and calculates the threshold that minimizes the intra-class variance.

\section{Feature Extraction}

Four algorithms were implemented in order to extract the features described in the ABCD rule (see Table I). The asymmetry analysis was made by computing the principal and secondary axes of inertia, as described in the work of Celebi et al. [11]. The axes of the image are aligned with the axes of inertia, which allows a better assessment of the lesion symmetry in terms of geometry, internal structures and color distribution. The border analysis aims to find abrupt ending of pigment pattern at the periphery of the lesion, so we defined two peripheral regions, one inside and other outside the lesion using the Euclidian Distance Transform, as suggested in [11]. In order to find the six colors proposed by the ABCD rule, the implemented color detection algorithm consists in two stages: color clustering using a mean-shift algorithm [12] and color supervised classification based on a dataset of reference RGB codes. Finally, the system performs the detection of 5 types of differential structures relevant for the detection of mela- noma: homogeneous areas, streaks, dots, globules and pigment network. The implemented methodology is based on texture, structural and connected component analysis [13].

TABLE 1.

METRICS FOR THE ABCD FEATURES.

\begin{tabular}{|c|c|c|c|}
\hline $\begin{array}{c}\text { ABCD } \\
\text { Feature }\end{array}$ & $\begin{array}{c}\text { Number of } \\
\text { metrics }\end{array}$ & $\begin{array}{c}\text { Color } \\
\text { spaces }\end{array}$ & Description \\
\hline Asymmetry & 102 & $\begin{array}{c}\text { RGB, } \\
\text { HSV, } \\
\text { CIELAB }\end{array}$ & $\begin{array}{c}34 \text { metrics for each } \\
\text { color space (17 for } \\
\text { each axis). }\end{array}$ \\
\hline Border & 144 & $\begin{array}{c}\text { RGB, } \\
\text { HSV, } \\
\text { CIELAB }\end{array}$ & $\begin{array}{c}48 \text { metrics for each } \\
\text { color space }(8 \text { for each } \\
\text { segment). }\end{array}$ \\
\hline Color & 12 & RGB & $\begin{array}{c}\text { metrics for each } \\
\text { color. }\end{array}$ \\
\hline $\begin{array}{c}\text { Differential } \\
\text { Structures }\end{array}$ & 28 & RGB & $\begin{array}{c}\text { 6 metrics for each } \\
\text { differential structure. }\end{array}$ \\
\hline
\end{tabular}

\section{Feature Selection}

Before training the classifiers, a feature selection step takes place using the Principal Component Analysis (PCA) method [14]. The PCA is a statistical method that uses an orthogonal transformation to convert a set of observations of possibly correlated variables into a set of values of linearly uncorrelated variables called principal components. So, PCA was used to find the relevant metrics that better describes each specific criterion before training the classifiers.

\section{E. Supervised Classification}

The supervised classification of each ABCD criteria is made separately. To train the classifiers we use a dataset of 504 images (214 suspicious lesions and 290 benign lesions) manually classified according to the ABCD rule by a specialist from the Portuguese Institute of Oncology of Porto (IPO-Porto). The classification of each ABCD criteria is made separately using random trees classifiers, which are trained with the correspondent extracted metrics. As a result, we will obtain the score for each criterion, as stated in the ABCD rule.

\section{MOBILE APPLICATION}

\section{A. Information Architecture}

In the proposed patient-oriented solution, the information flows along three main groups: users, skin lesions and check-ups (Figure 1). The application may have several users; one user may have several skin lesions; one skin lesion has one or more check-ups. Each user has the following information: 1) name; 2) gender; and 3) age. Each skin lesion has the following information: 1) location on the body; and 2) list of check-ups. Each check-up has the following information: 1) skin lesion image; 2) check-up date; 3 ) four ABCD features scores (generated by the IPM); and 4) skin lesion size (in $\mathrm{mm}$ ). 


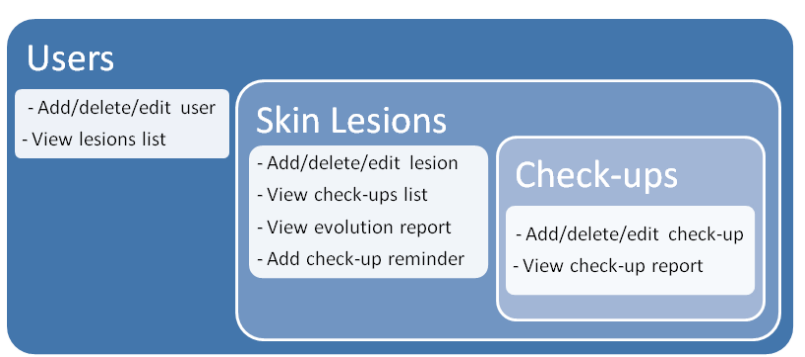

Figure 1. Schematization of the information architecture and system functionalities.

\section{B. Application Features}

Some of the main features of the developed application are: 1) Capture images with the smartphone built-in camera or load images from the mobile gallery; 2) Send the images to the server for analysis; 3) Receive real time visual feedback of the extracted ABCD features (Figure 2.d); 4) Receive an analysis report (Figure 2.b); 5) Save the analysis on the local database; 6) View list of skin lesions and check-ups (Figure 2.c);

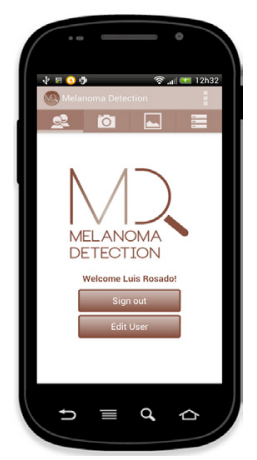

(b)

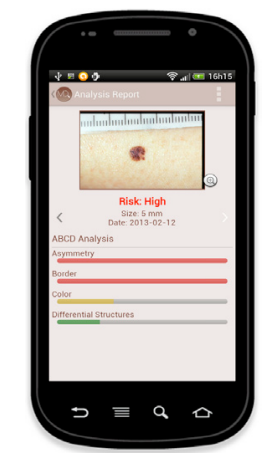

(b)

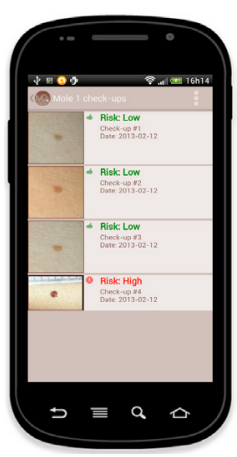

(c)

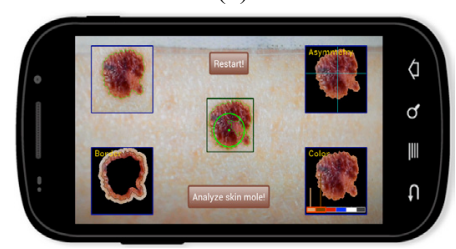

(d)

Figure 2. Mobile application screenshots: (a) Main menu; (b) Analysis report of a specific check-up; (c) Check-ups list of a specific skin lesion;

(d) Real time visual feedback of the extracted ABCD features.

\section{CONCLUSIONS AND FUTURE WORK}

This paper presents a prototype of a mobile-based system for skin lesion analysis with patient-oriented features and functionalities, using supervised classification. As future work, from the image processing perspective one aims to develop a version of the application that allows the IPO-Porto doctors to test it in real patients and input if they agree with the automatic analysis. This way we will be able to quantify the correlation between the automatic and dermatologist classification. We are also looking forward to conduct usability tests to the developed application, as well as explore the capability of capturing images with dermoscopic quality using the smartphone camera, for instance using adaptable dermoscopes for smartphones.

\section{REFERENCES}

[1] "World Health Organization." [Online]. Available: http://www.who.int/. [Last access: August 2013].

[2] Unidade Operacional Planeamento e Investimentos, Unidade Funcional Estudos e Planeamento de Recursos Humanos, "Actuais e Futuras Necessidades Previsionais de Médicos (SNS)”, 2011.

[3] W. Stolz, A. Riemann, AB. Cognetta. "ABCD rule of dermatoscopy: a new practical method for early recognition of malignant melanoma", Eur J Dermatol, 1994, Vol. 4, pp. 521-527.

[4] E. A. Krupinski, B. LeSueur, L. Ellsworth, N. Levine, R. Hansen, N. Silvis, P. Sarantopoulos, P. Hite, J. Wurzel, R. S. Weinstein, and others, "Diagnostic accuracy and image quality using a digital camera for teledermatology", Telemedicine Journal, vol. 5, no. 3, pp. 257-263, 1999. http://dx.doi.org/10.1089/107830299312005

[5] C. Massone, R. Hofmann-Wellenhof, V. Ahlgrimm-Siess, G. Gabler, C. Ebner, and H. P. Soyer, "Melanoma screening with cellular phones", PloS one, vol. 2, no. 5, p. e483, 2007. http://dx.doi.org/10.1371/journal.pone.0000483

[6] S. Q. Wang, H. Rabinovitz, A. W. Kopf, and M. Oliviero "Current technologies for the in vivo diagnosis of cutaneous melanomas", Clinics in dermatology, 22:217-222, 2004. http://dx.doi.org/10.1016/j.clindermatol.2003.12.008

[7] Jersey, the open source JAX-RS Reference Implementation for building RESTful Web services. [Online] http://jersey.java.net/. [Last accessed: August 2013].

[8] OpenCV (Open Source Computer Vision), open source library of programming functions for real time computer vision. [Online] http://opencv.willowgarage.com/. [Last accessed: August 2013].

[9] G. Schaefer, M. I. Rajab, M. E. Celebi, and H. Iyatomi. "Colour and Contrast Enhancement for Improved Skin Lesion Segmentation", Computerized Medical Imaging and Graphics, 2011, Vol. 35(2), pp. 99-104. http://dx.doi.org/10.1016/j.compmedimag.2010.08.004

[10] Otsu N. "A threshold selection method from gray-level histograms", IEEE Transactions on Systems, Man, and Cybernetics, 1979, Vol. 9(1). pp. 62-66. http://dx.doi.org/10.1109/TSMC.1979. $\underline{4310076}$

[11] M. E. Celebi, H. Iyatomi, et al. "Automatic Detection of BlueWhite Veil and Related Structures in Dermoscopy Images", Computerized Medical Imaging and Graphics, 2008, Vol. 32(8), pp. 670-677. http://dx.doi.org/10.1016/j.compmedimag.2008.08.003

[12] D. Comaniciu, P. Meer, "Distribution Free Decomposition of Multivariate Data", Pattern Analysis and Applications, 1999, Vol. 2(1), pp. 22-30. http://dx.doi.org/10.1007/s100440050011

[13] L. Rosado, "Automatic Detection of Differential Structures in Dermoscopic Images", Proceedings of 17th Portuguese Conference on Pattern Recognition, 2011, pp. 42-44.

[14] Shifei Ding, Hong Zhu, Weikuan Jia, Chunyang Su: A survey on feature extraction for pattern recognition. Artif. Intell. Rev., 2012, pp. 169-180. http://dx.doi.org/10.1007/s10462-011-9225-y

\section{AUTHORS}

Luís Rosado is in the Research and Development Department of Fraunhofer Portugal AICOS, Porto, Portugal (e-mail: luis.rosado@fraunhofer.pt).

Maria João Vasconcelos is in the Research and Development Department of Fraunhofer Portugal AICOS.

Márcia Ferreira is in the Dermatology Department of the Portuguese Institute of Oncology of Porto, Porto, Portugal.

This work was supported by FCT in the scope of project PTDC/EBB$\mathrm{BMD} / 3088 / 2012$. This article is an extended and modified version of a paper presented at the International Conference exp.at'13, held 18-20 September 2013, in Coimbra, Portugal. Submitted 18 November 2013. Published as re-submitted by the authors 04 December 2013. 